\title{
Medial Patella Subluxation after Minor Trauma
}

\author{
Seung Hyun Hwang*, Chang Hyun Nam, Kyung Won Choi, Hye Sun Ahn \\ Joint \& Arthritis Research, Department of Orthopaedic Surgery, Himchan Hospital, Seoul, Korea \\ Email: *hsh3182@naver.com
}

How to cite this paper: Hwang, S.H., Nam, C.H., Choi, K.W. and Ahn, H.S. (2017) Medial Patella Subluxation after Minor Trauma. Open Journal of Orthopedics, 7, 295-300.

https://doi.org/10.4236/ojo.2017.710029

Received: September 1, 2017

Accepted: September 27, 2017

Published: September 30, 2017

Copyright (@) 2017 by authors and Scientific Research Publishing Inc. This work is licensed under the Creative Commons Attribution International License (CC BY 4.0).

http://creativecommons.org/licenses/by/4.0/

\begin{abstract}
Most cases of medial patella subluxation occur under iatrogenic conditions that are often associated with a prior excessive lateral retinacular release procedure. We report a case of medial subluxation following minor trauma with no history of previous lateral release. The abnormality was identified on magnetic resonance imaging and was successfully treated by direct repair of the lateral retinaculum. The recognition and treatment of medial subluxation of the patella without retinacular release can be difficult. Therefore, consideration of multiple causes through careful medical history taking, physical examination, and radiologic examination is needed for accurate diagnosis.
\end{abstract}

\section{Keywords}

Medial Patella Subluxation, Arthroscopy, Lateral Retinacular, Ligament Reconstruction

\section{Introduction}

Lateral subluxation or dislocation of the patella is a well-recognized orthopedic disorder, but medial patellar subluxation (MPS) is poorly recognized [1] [2]. Most MPS is iatrogenic and associated with excessive lateral retinacular release procedures [3] [4] [5] [6]. However, there is limited literature describing traumatic and isolated MPS in the absence of a previous lateral release [7] [8]. We report a case of medial subluxation following minor trauma that was demonstrated by magnetic resonance imaging (MRI) and successfully treated by direct repair of the lateral retinaculum.

\section{Case}

This study was approved by the institutional review board of our hospital, and patient provided informed consent. A 35-year-old female patient presented to our outpatient clinic complaining of aggravating lateral knee pain. She has no 
specific history of medical problem and surgery of patellofemoral component. She had sustained an injury after slipping and falling and had been diagnosed with hemarthrosis and knee contusion at a local clinic six months prior to presentation. She had been treated conservatively with a patella-stabilizing brace for one month and with quadriceps muscle training. Additionally, she underwent multiple joint aspirations for joint effusion, but the lateral knee pain and swelling gradually worsened.

On physical examination at the time of visit, she had a full range of motion of the right knee with painful terminal flexion, and there was tenderness to palpation at the lateral border of the patella. There was moderate joint effusion and a palpable lateral defect that corresponded to a mass-like soft tissue swelling at the lateral border of the patella. She had a positive medial patellar apprehension test in which she experienced the patella being subluxated with a medially directed force on the lateral border of the patella. The "gravity subluxation test" described by Nonwiler et al. was not used [9].

Radiographs of her knee revealed dysplastic medial and lateral condyles and a dysplastic trochlea (Figure 1). Stress radiography was not used.

MRI of the knee was performed in order to observe the patellar retinaculum. Axial images of the MRI showed a slight medial tilt of patella, a defect in the lateral retinaculum, and moderate joint fluid. Sagittal images showed both an abnormal anterior crucial ligament (ACL) and posterior crucial ligament (PCL) with mucoid degeneration (Figure 2).

Based on these findings, arthroscopy with open lateral retinacular closure was planned. Diagnostic arthroscopy was performed using standard anteromedial and anterolateral portals. There was no meniscal pathology medially or laterally. Both the ACL and PCL were intact, but they no were longer enveloped by the synovium and showed yellowish, hypertrophic changes. The hypertrophic ACL and PCL filled the entire intercondylar notch, causing impingement. Partial excision of the ACL and PCL was performed to relieve the impingement (Figure 3). Then, patellar tracking was viewed from both the anterolateral and anteromedial portals. This demonstrated normal articular surfaces and a deficient lateral retinaculum. Lateral patellar laxity was present with the knee fully extended (Figure 4). After the arthroscopic procedure, a $3 \mathrm{~cm}$ skin incision was made on the thin, inflated, palpable defect area in the lateral retinaculum. The subcutaneous tissue was dissected to the level of the inflated joint capsule, and the defect was identified. The lateral retinacular defect was closed with absorbable sutures with the knee flexed at $20^{\circ}$. After closure, the patency of the knee was confirmed with arthroscopy, and the portals were closed.

The knee was immobilized in a brace for weight-bearing activity for four weeks. A gradual increase to full weight-bearing activity without a brace was permitted. At the 12 month follow-up appointment, the patient exhibited full quadriceps and hamstring strength. She denied instability symptoms, and her knee pain had improved. 


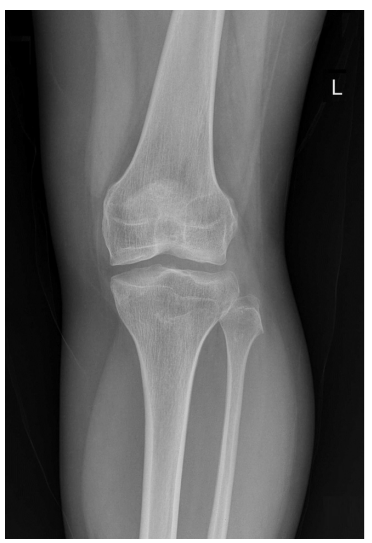

(a)

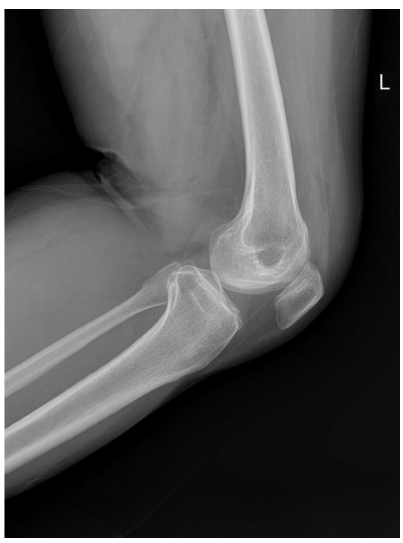

(b)

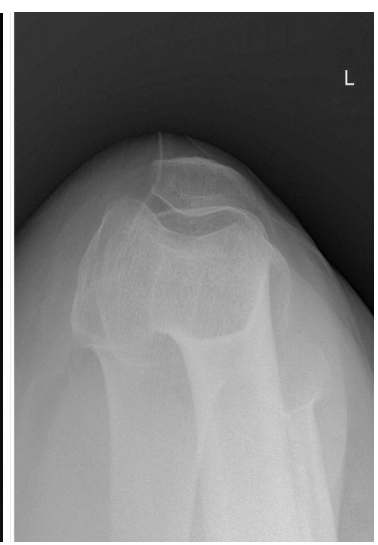

(c)

Figure 1. Anteroposterior (a); lateral (b); and merchant (c) radiographs of the right knee joint show a dysplastic medial (white arrow) and lateral (black arrow) condyle and a lack of patellar tilt.

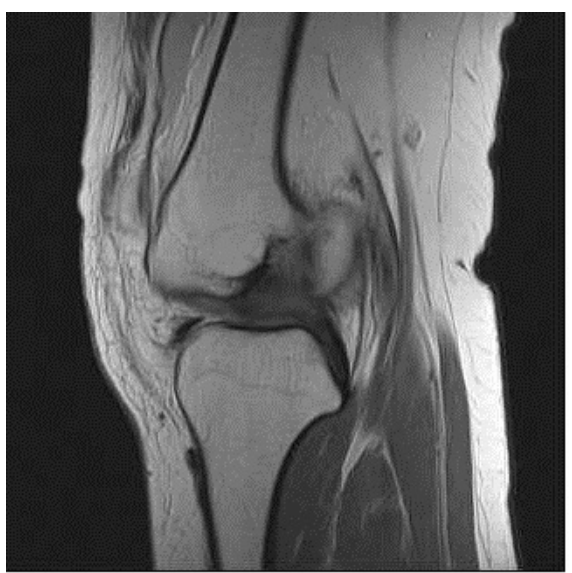

(a)

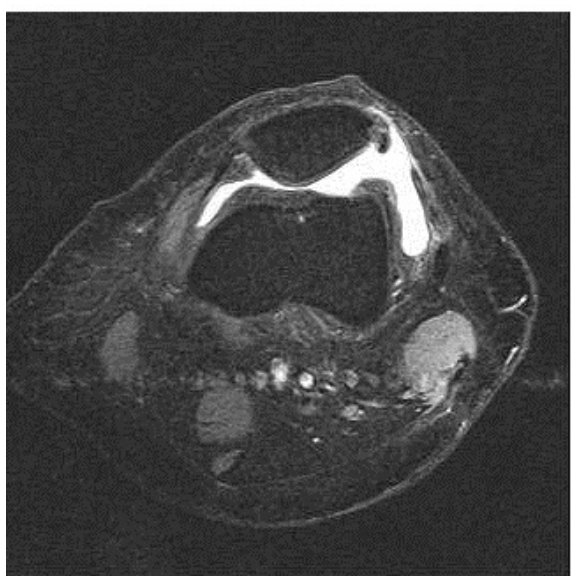

(c)

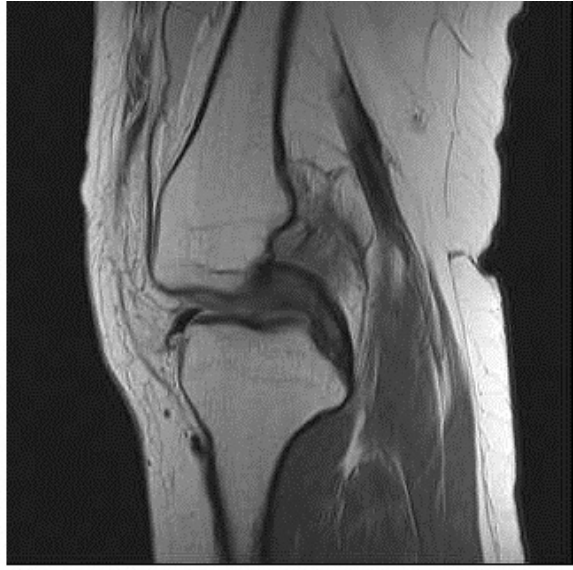

(b)

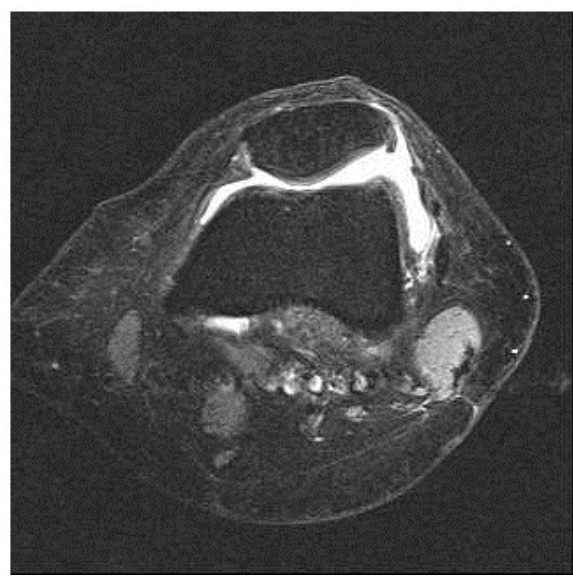

(d)

Figure 2. Sagittal images of anterior (a) and posterior (b) cruciate ligaments on magnetic resonance imaging show homogeneous intensity and hypertrophy. Axial image (c) (d) shows a slight medial patellar tilt and a defect of the lateral patella retinaculum (white arrow) (T1-weighted (a) (b) and proton density-weighted (c) (d)). 


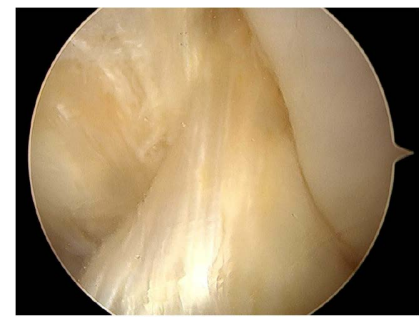

(a)

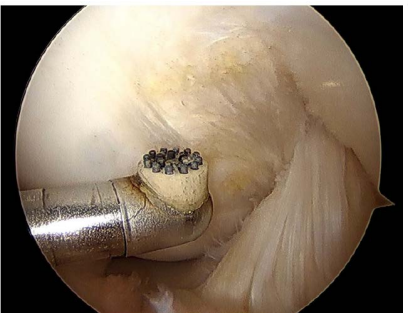

(c)

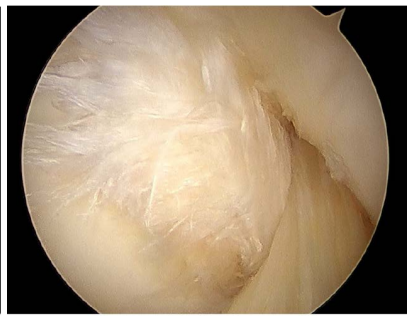

(b)

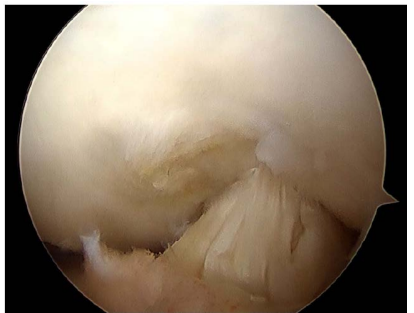

(d)

Figure 3. Arthroscopic findings of the anterior cruciate ligament (a) and posterior cruciate ligament (b) show preoperative hypertrophic changes. The postoperative state after partial excision is also shown (c) (d).

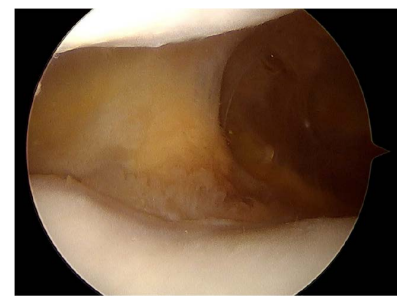

(a)

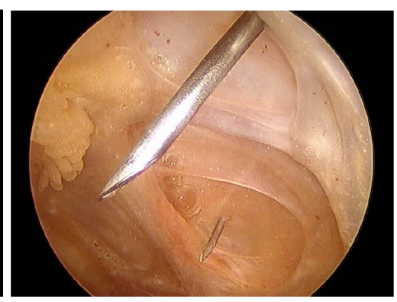

(b)

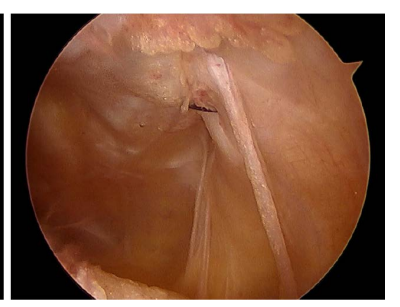

(c)

Figure 4. Arthroscopic view of the patellofemoral articulation shows significant tilting of the patella and lateral laxity at full knee extension and at $30^{\circ}$ of knee flexion (a). Arthroscopic view of the lateral si The postoperative state after closure of the lateral retinaculum is also shown (c). de of the patella shows a preoperative defect of the lateral retinaculum (b) needle point.

\section{Discussion}

Almost all cases of medial patella subluxation are caused by extensive release or over-release of the lateral retinaculum that was intended to relieve anterior knee pain or patellar instability [3] [4] [5] [6]. There is limited literature describing isolated or spontaneous MPS in the absence of a previous lateral release [7] [8].

MPS was first described in 1987 by Betz et al. as a complication of lateral retinacular release [10]. Since then, Hughston et al. reported medial subluxation in $50 \%$ of knees with recurrent patellar dislocation in which arthroscopic lateral retinacular release was performed [11]. Shellock et al. reported medial subluxation in 13 of 14 knees (93\%) with recurrent patellar dislocation in which lateral retinacular release was performed [12].

The most common findings of MPS after lateral retinacular release are anterolateral knee pain, tenderness, and a palpable defect at the focal site of a pre- 
vious lateral retinacular release. Additionally, pain and apprehension are noted when medial stress is applied to the patella. Isolated MPS has similar clinical findings except that there are no previous surgical scars suggestive of lateral retinacular release. In our patient, there was no previous surgical history; however, she presented with joint effusion, tenderness to palpation, a palpable defect on the lateral side of the patella, and a positive medial apprehension test on physical examination. In the present case, noting aggravating symptoms after a previous minor trauma was helpful in the diagnosis. We believe that a lateral retinacular tear might have occurred with the trauma, and it gradually progressed into a large lateral retinacular defect, which is similar to the complication of a failed lateral retinacular release procedure.

Various surgical techniques have been used to treat MPS including open retinacular closure, lateral retinaculum imbrication, lateral retinaculum reconstruction, and lateral patellofemoral ligament reconstruction [3] [8] [13] [14]. We chose an open lateral retinacular closure, which is a form of direct repair focused on reapproximating the lateral retinacular tissue without the need for more invasive lateral patellofemoral ligament reconstruction. Postoperatively, medial dislocation of patella did not occur, and knee pain improved.

If a patient is not acutely aware of patellar dislocation and has no previous surgical history, the recognition of isolated MPS can be difficult. Therefore, a high index of suspicion, careful medical history taking, physical examination, and radiologic examination is needed for accurate diagnosis.

\section{References}

[1] Colvin, A.C. and West, R.V. (2008) Patellar Instability. The Journal of Bone and Joint Surgery, American Volume, 90, 2751-2762. https://doi.org/10.2106/JBJS.H.00211

[2] Aglietti, P., Buzzi, R., De Biase, P. and Giron, F. (1994) Surgical Treatment of Recurrent Dislocation of the Patella. Clinical Orthopaedics and Related Research, 308, 8-17.

[3] Heyworth, B.E., Carroll, K.M., Dawson, C.K. and Gill, T.J. (2012) Open Lateral Retinacular Closure Surgery for Treatment of Anterolateral Knee Pain and Disability after Arthroscopic Lateral Retinacular Release. The American Journal of Sports Medicine, 40, 376-382. https://doi.org/10.1177/0363546511428600

[4] Sanchis-Alfonso, V. and Merchant, A.C. (2015) Iatrogenic Medial Patellar Instability: An Avoidable Injury. Arthroscopy, 31, 1628-1632. https://doi.org/10.1016/j.arthro.2015.01.028

[5] Sanchis-Alfonso, V., Montesinos-Berry, E., Monllau, J.C. and Merchant, A.C. (2015) Results of Isolated Lateral Retinacular Reconstruction for Iatrogenic Medial Patellar Instability. Arthroscopy, 31, 422-427. https://doi.org/10.1016/j.arthro.2014.09.005

[6] Udagawa, K., Niki, Y., Matsumoto, H., Matsumoto, H., Enomoto, H., Toyama, Y. and Suda, Y. (2014) Lateral Patellar Retinaculum Reconstruction for Medial Patellar Instability Following Lateral Retinacular Release: A Case Report. Knee, 21, 336-339. https://doi.org/10.1016/j.knee.2013.04.011

[7] Saper, M.G. and Shneider, D.A. (2014) Medial Patellar Subluxation without Pre- 
vious Lateral Release: A Case Report. Journal of Pediatric Orthopaedics B, 23, 350-353. https://doi.org/10.1097/BPB.0000000000000054

[8] Richman, N.M. and Scheller Jr., A.D. (1998) Medial Subluxation of the Patella without Previous Lateral Retinacular Release. Orthopedics, 21, 810-813.

[9] Nonweiler, D.E. and De Lee, J.C. (1994) The Diagnosis and Treatment of Medial Subluxation of the Patella after Lateral Retinacular Release. The American Journal of Sports Medicine, 22, 680-686. https://doi.org/10.1177/036354659402200517

[10] Betz, R.R., Magill, J.T. and Lonergan, R.P. (1987) The Percutaneous Lateral Retinacular Release. The American Journal of Sports Medicine, 15, 477-482. https://doi.org/10.1177/036354658701500508

[11] Hughston, J.C. and Deese, M. (1988) Medial Subluxation of the Patella as a Complication of Lateral Retinacular Release. The American Journal of Sports Medicine, 16, 383-388. https://doi.org/10.1177/036354658801600413

[12] Shellock, F.G., Mink, J.H., Deutsch, A.L. and Fox, J.M. (1989) Patellar Tracking Abnormalities: Clinical Experience with Kinematic MR Imaging in 130 Patients. Radiology, 172, 799-804. https://doi.org/10.1148/radiology.172.3.2772191

[13] Hughston, J.C., Flandry, F., Brinker, M.R., Terry, G.C. and Mills, J.C. (1996) Surgical Correction of Medial Subluxation of the Patella. The American Journal of Sports Medicine, 24, 486-491. https://doi.org/10.1177/036354659602400413

[14] Teitge, R.A. and Torga Spak, R. (2004) Lateral Patellofemoral Ligament Reconstruction. Arthroscopy, 20, 998-1002. https://doi.org/10.1016/S0749-8063(04)00798-4 